\title{
Increased HOXC6 expression predicts chemotherapy sensitivity in patients with esophageal squamous cell carcinoma
}

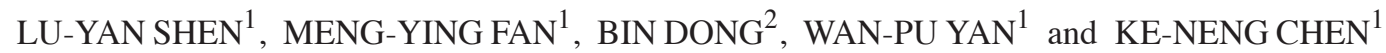 \\ Departments of ${ }^{1}$ Thoracic Surgery I and ${ }^{2}$ Pathology, Key Laboratory of Carcinogenesis and Translational Research \\ (Ministry of Education), Peking University Cancer Hospital and Institute, Beijing 100142, P.R. China
}

Received May 19, 2016; Accepted March 10, 2017

DOI: $10.3892 / 01.2017 .6772$

\begin{abstract}
Increased expression of homeobox C6 (HOXC6) predicts poor prognosis of patients with esophageal squamous cell carcinoma (ESCC) and promotes ESCC cell proliferation. Additionally, the expression of HOXC6 was upregulated in chemosensitive ESCC cell lines. Therefore, it was hypothesized that HOXC6 may be associated with chemosensitivity of ESCC. Patients with ESCC who underwent neoadjuvant chemotherapy followed by surgery by a single-surgeon team between January 2000 and December 2012 were enrolled in the present study. Pretreatment biopsy specimens and postoperative resection samples were collected. Immunohistochemistry was performed to examine HOXC6 expression, and the association between HOXC6 expression and tumor regression grade (TRG) was analyzed. In cell lines exhibiting stable knockdown of HOXC6, Cell Counting Kit- 8 assays were used to evaluate the chemosensitivity of cells to various concentrations of cisplatin and paclitaxel. A total of 51 pretreatment biopsy specimens were assessed, and patients with increased expression of HOXC6 in pretreatment biopsy specimens exhibited higher TRGs. A total of 186 surgical samples were evaluated; HOXC6 was expressed at a decreased level in patients with higher TRG and at a high level in patients with lower TRG. In addition, downregulation of HOXC6 decreased the sensitivity of ESCC cell lines to cisplatin and paclitaxel, resulting in an increased half-maximal inhibitory concentration. Increased expression of HOXC6 prior to treatment was associated with chemosensitivity in ESCC tissues.
\end{abstract}

Correspondence to: Dr Ke-Neng Chen, Department of Thoracic Surgery I, Key Laboratory of Carcinogenesis and Translational Research (Ministry of Education), Peking University Cancer Hospital and Institute, 52 Fucheng Road, Haidian, Beijing 100142, P.R. China

E-mail: chenkeneng@bjmu.edu.cn

Key words: esophageal squamous cell carcinoma, homeobox C6, chemotherapy response, chemosensitivity, cisplatin, paclitaxel, tumor regression grade

\section{Introduction}

Esophageal cancer is a common malignancy in China, and surgery is currently the primary treatment modality. The efficacy of surgery is associated with the tumor-node-metastasis staging of the tumor. For patients with early-stage esophageal cancer, surgery may provide strong local control; however, in patients with locally advanced cancer, the benefits of surgery are limited (1). In previous studies, comprehensive treatments, particularly those including neoadjuvant therapy, have been identified to improve the prognosis of patients with locally advanced cancer $(2,3)$. However, previous studies have demonstrated that the improved efficacy associated with neoadjuvant therapy is limited to patients who respond to chemotherapy, and the prognosis of non-responders is worse compared with that of patients who received surgery alone $(2,4)$. Thus, prediction of the efficacy of neoadjuvant chemotherapy prior to treatment and identifying useful predictive biomarkers is required.

The highly conserved homeobox $(H O X)$ gene family serves an important function in embryonic development; it is responsible for encoding transcription factors that regulate cell proliferation and differentiation. The expression of $H O X$ genes strictly follows the principle of temporal and spatial collinearity. Therefore, the expression patterns of $H O X$ genes vary according to the type of tissue and the developmental stage $(5,6)$. Alterations in the expression patterns of $H O X$ gene family members cause dysregulation of HOX protein function, further leading to unbalanced cell proliferation and differentiation; this is hypothesized to contribute to tumorigenesis. In the last two decades, studies have identified that the expression patterns of HOX genes are abnormal in a variety of tumors, including esophageal cancer (7-11). Our previous studies $(8,10)$ demonstrated that HOXC6 serves a key function in esophageal squamous cell carcinoma (ESCC) and is abnormally expressed in ESCC tissues. Single-surgery patients with increased expression of HOXC6 exhibited a significantly shorter median survival time compared with patients with decreased expression of HOXC6. Additionally, we previously demonstrated with in vitro study that cell strains with decreased expression of HOXC6 exhibit markedly lower proliferation capacity, and higher apoptosis rates, compared with cell lines with increased expression of HOXC6 (unpublished data). We previously applied gene expression profiling to analyze the gene expression 
difference between chemosensitive and chemoresistant cell lines and found that cells exhibiting high HOXC6 expression are more sensitive to cisplatin and paclitaxel (12). Therefore, it is hypothesized that HOXC6 may be involved in mediating chemosensitivity in patients with ESCC. In the present study, the association between HOXC6 expression in ESCC tissues from patients who received neoadjuvant chemotherapy and the pathological indicator of tumor regression grade (TRG), which reflects chemoresponsiveness, were investigated. The applicability of HOXC6 as a biomarker for the prediction of neoadjuvant chemotherapy outcomes was also assessed and the results were validated in vitro.

\section{Materials and methods}

Patients and specimens. In total 224 patients who underwent neoadjuvant chemotherapy and esophagectomy by a single-surgeon team between January 2000 and December 2012, at the Department of Thoracic Surgery, Peking University Cancer Hospital (Beijing, China) were enrolled in the present study. Of the 224 patients, 176 were male, 48 were female and the mean age was 59.7 years. A total of 51 cases of formalin-fixed paraffin-embedded blocks of pretreatment biopsies and 186 cases of resected specimens were collected. The present study was approved by the Ethics and the Academic Committees of Peking University School of Oncology (Beijing, China) and informed verbal consent was obtained from all patients.

Neoadjuvant chemotherapy. A platinum-based two-drug combination, primarily paclitaxel/cisplatin at a proportion of 95 and 5\% was 5-FU/cisplatin, was used in neoadjuvant chemotherapy. On day 1 , paclitaxel, at a dose of $175 \mathrm{mg} / \mathrm{m}^{2}$ of body surface area, was administered intravenously. On days $1-3$, cisplatin, at a dose of $25 \mathrm{mg} / \mathrm{m}^{2}$ of body surface area, was administered intravenously and a single cycle of treatment lasted 21 days. Enhanced chest computed tomography (CT) and esophagography were used to evaluate the curative effects of the treatment. Between 1 and 4 cycles of neoadjuvant chemotherapy were administered prior to surgery.

Tumor regression grade assessment. All enrolled subjects were reviewed again by two experienced pathologists who were blinded to the clinical information and gene expression. Tumors were graded using the TRG; a four-point scale based on the histological tumor response assessment, described by Mandard et al (13). This assessment was defined as: Grade I, no residual tumor cells; grade II, almost complete response with $<10 \%$ vital residual tumor cells (VRTCs); grade III, 10-50\% VRTCs; and grade IV, $>50 \%$ VRTCs. In the present study, histological tumor response was classified as major (TRG1/2/3) or minor (TRG4) histopathological tumor response.

Cell lines and cell culture. Human ESCC EC109 cells were obtained from the Cancer Hospital of the Chinese Academy of Medical Science (Beijing, China). Human ESCC KYSE 150, KYSE410, KYSE450 and KYSE510 cells were purchased from the Japanese Collection of Research Bioresources cell bank (Osaka, Japan). Cells were cultured in RPMI-1640 medium (Hyclone; GE Healthcare, Logan, UT, USA) with $10 \%$ heat-inactivated fetal bovine serum at $37^{\circ} \mathrm{C}$ in a humidified atmosphere containing $5 \% \mathrm{CO}_{2}$.

A cellular model for stable HOXC6 knockdown was estab-

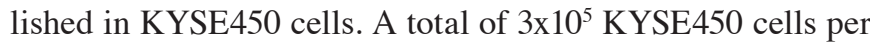
well were plated in a 6-well plate and were grown overnight, in the conditions previously described, to $80 \%$ confluence. Then the cells were transfected with $100 \mu 1$ medium containing either short hairpin RNA (shRNA; lentivirus-plasmid-mediated) against HOXC6 (sequence: GGACATAACACCAGACC TCA) or scrambled shRNA, in the Pglv3/GFP+puro vector (all from Shanghai GenePharma Co., Ltd., Shanghai, China). Stably transfected cells were selected with $2 \mu \mathrm{g} / \mathrm{ml}$ puromycin (Amresco, LLC, Solon, OH, USA).

Reverse transcription-quantitative polymerase chain reaction (RT-qPCR). Total RNA from EC109, KYSE150, KYSE410, KYSE450 and KYSE510 cells was extracted with Trizol (Invitrogen; Thermo Fisher Scientific Inc., Waltham, MA, USA) according to the manufacture's protocol and $1 \mu \mathrm{g}$ RNA was reverse-transcribed using the RevertAid First Strand cDNA Synthesis kit (Thermo Fisher Scientific Inc.). The reverse transcription reaction was performed sequentially for $60 \mathrm{~min}$ at $42^{\circ} \mathrm{C}$ and for $5 \mathrm{~min}$ at $70^{\circ} \mathrm{C}$. qPCR was performed using Power SYBR-Green PCR Master Mix (Thermo Fisher Scientific Inc.). PCR runs and fluorescence detection were performed in a Rotor-Gene 6000 Real-Time PCR system (Applied Biosystems; Thermo Fisher Scientific, Inc.). The sequences of the PCR primers were as follows: HOXC6 forward, 5'-CACCGCCTATGATCCAGTGAGGCA-3' and reverse, 5'-GCTGGAACTGAACACGACATTCTC-3'; GAPDH forward, 5'-CTCTGACTTCAACAGCGACACC-3'; and reverse, 5'-CTGTTGCTGTAGCCAAATTCGTT-3'. The cycling conditions were as follows: $95^{\circ} \mathrm{C}$ for $10 \mathrm{~min}$ followed by 40 cycles of $95^{\circ} \mathrm{C}$ for $20 \mathrm{sec}, 58^{\circ} \mathrm{C}$ for $20 \mathrm{sec}$ and $70^{\circ} \mathrm{C}$ for $20 \mathrm{sec}$. GAPDH was used as an internal control. The $2^{-\Delta \Delta \mathrm{Cq}}$ method was used to determine the RNA expression level (14). All experiments were performed in triplicate.

Western blotting. The proteins were extracted by using radioimmunoprecipitation lysis buffer and separated by $10 \%$ SDS-PAGE with $30 \mu \mathrm{g}$ protein per lane and transferred onto a polyvinylidene fluoride membrane, followed by western blot analysis. The membrane was blocked using 5\% bovine serum albinum at room temperature for $1 \mathrm{~h}$. It was then immunoreacted with rabbit anti-HOXC6 polyclonal antibody (dilution, 1:300; \#ab151575; Abcam, Cambridge, UK) as primary antibody. Goat anti-rabbit polyclonal horseradish peroxidase-conjugated IgG was used as a secondary antibody. Immunoreactivity was detected using an enhanced chemiluminescence reaction kit. As a loading control, GADPH was detected using a goat polyclonal antibody (dilution, 1:2,000; \#ab9845; Abcam). All experiments were performed in triplicate.

Cell Counting Kit-8 (CCK-8) assay. Cells were plated in 96-well plates at a density of 10,000 cells/well in $100 \mu \mathrm{l}$ complete medium and incubated overnight at $37^{\circ} \mathrm{C}$. The cells were then treated with gradient dilution of cisplatin $(0.2,0.4,0.8,1.6,3.2,6.4,12.8$ and $25.6 \mu \mathrm{g} / \mathrm{ml})$ and paclitaxel $(0.002,0.008,0.032,0.128,0.512$, $2.048,8.192$ and $32.768 \mu \mathrm{M})$ in $100 \mu \mathrm{l}$ complete medium. After $48 \mathrm{~h}$ of incubation, $100 \mu \mathrm{l}$ CCK-8 reagent (Dojindo Molecular 
A

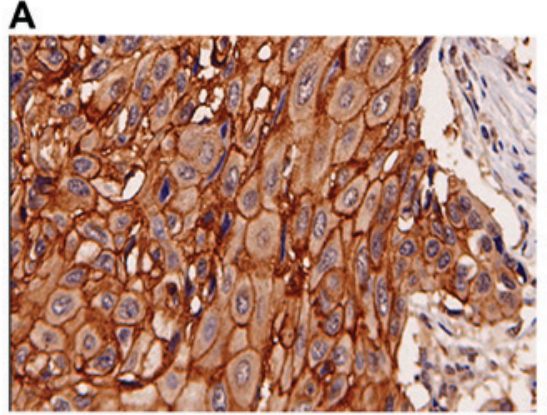

B

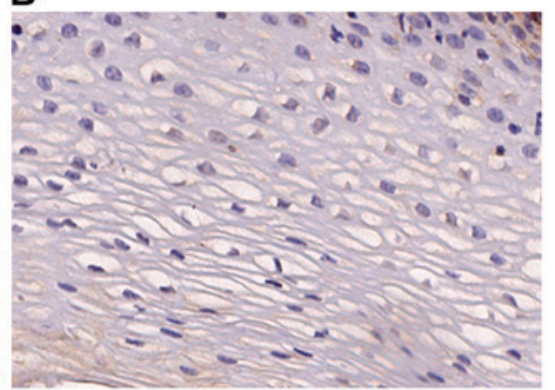

Figure 1. Expression of HOXC6 protein in ESCC. (A) Increased expression of HOXC6 in ESCC (magnification, x200). (B) Negative expression of HOXC6 in normal esophageal epithelium (magnification, x200). HOXC6, homeobox C6; ESCC, esophageal squamous cell carcinoma.

Table I. Association of HOXC6 expression in pretreatment biopsies TRG $(\mathrm{n}=51)$.

\begin{tabular}{lcrr}
\hline $\begin{array}{l}\text { HOXC6 expression } \\
\text { (endoscope) }\end{array}$ & $\begin{array}{c}\text { TRG1/2/3, } \\
\mathrm{n}(\%)\end{array}$ & $\begin{array}{c}\text { TRG4, } \\
\mathrm{n}(\%)\end{array}$ & P-value \\
\hline Low & $6(18.8)$ & $9(47.4)$ & 0.030 \\
High & $26(81.3)$ & $10(52.6)$ & \\
\hline
\end{tabular}

HOXC6, homeobox C6; TRG, tumor regression grade.

Table II. Association of HOXC6 expression in postoperative specimens and TRG $(\mathrm{n}=164)$.

\begin{tabular}{lccr}
\hline $\begin{array}{l}\text { HOXC6 expression } \\
\text { (endoscope) }\end{array}$ & $\begin{array}{c}\text { TRG2/3, } \\
\mathrm{n}(\%)\end{array}$ & $\begin{array}{c}\text { TRG4, } \\
\mathrm{n}(\%)\end{array}$ & P-value \\
\hline Low & $41(62.1)$ & $25(37.9)$ & 0.005 \\
High & $39(39.8)$ & $59(60.2)$ & \\
\hline
\end{tabular}

TRG1 was defined as 'no residual tumor cells', so TRG1 samples were not included in this cohort of resected specimens. HOXC6, homeobox C6; TRG, tumor regression grade.

Technologies Inc., Kumamoto, Japan) was added to each well. After $4 \mathrm{~h}$ of incubation at $37^{\circ} \mathrm{C}$, the absorbance of each well was measured at $450 \mathrm{~nm}$ using a VersaMax microplate reader (Molecular Devices, LLC, Sunnyvale, CA, USA).

Immunohistochemistry (IHC). The sections were deparaffinized in xylene and rehydrated using decreasing concentrations of ethanol (100, 95, 85 and 75\% ethanol). Following routine deparaffinization and rehydration, tissue sections were treated with $3 \% \mathrm{H}_{2} \mathrm{O}_{2}$ and then heated in citrate buffer ( $\mathrm{pH}$ 6.0) for antigen retrieval. The HOXC6 antigen-antibody reaction occurred overnight at $4^{\circ} \mathrm{C}$, following blocking with goat serum (ZSGB-Bio, Beijing, China). The streptavidin/peroxidase amplification kit (Zymed; Thermo Fisher Scientific, Inc.) was used to detect the signal of the HOXC6 antigen-antibody reaction. Peroxidase activity was developed with diaminobenzidine. All sections were counterstained with hematoxylin. Purified rabbit polyclonal antibody against human HOXC6 (cat no. \#ab151575; Abcam) was used at a dilution of 1:200, and biotin-conjugated goat anti-rabbit $\operatorname{IgG}$ was used as secondary antibody (dilution, 1:300; cat no. SPN-9001; ZSGB-Bio). Immunohistochemical signals were scored by two independent observers. The scores were calculated by multiplying the staining intensity and extent. The staining intensity was categorized by relative intensity as follows: 0 , negative; 1 , weak; 2 , moderate; and 3 , strong. Scores $<3$ were considered as low-level expression, whereas scores 3 were considered as high-level expression.

Statistical analysis. SPSS software (version 19.0; IBM SPSS, Armonk, NY, USA) was used to perform the statistical analysis. All in vitro experiments were performed at least three times with triplicates. When the data from different groups were compared, normal analysis and homogeneity of variance were checked first, and then an unpaired two-tailed Student's t-test analysis was used. Results are presented as the mean \pm standard deviation. The association between gene expression and TRG were evaluated using a $\chi^{2}$ test. $\mathrm{P}<0.05$ was considered to indicate a statistically significant difference.

\section{Results}

Increased HOXC6 expression is associated with tumor regression in patients with ESCC. To investigate the association between HOXC6 expression and chemoresistance, IHC was performed in 51 cases of patients with complete pretreatment biopsies and matched resected specimens. HOXC6 was overexpressed in ESCC cells compared with the normal epithelium (Fig. 1). HOXC6 expression in the pretreatment biopsies was associated with the response to chemotherapy in this set of patients with ESCC, but not resected specimens. The patients with high expression of HOXC6 in pretreatment biopsies achieved improved objective response to chemotherapy (TRG1/2/3). A total of 26 samples from patients sensitive to chemotherapeutic drugs exhibited significantly increased HOXC6 expression, and 9 cases from patients resistant to anticancer drugs exhibited decreased HOXC6 expression (Table I). To further investigate the association between HOXC6 expression in resected specimens and TRG, HOXC6 expression was examined in a larger cohort. The results revealed that in 164 cases of resected specimens (samples with TRG1 were not included, as TRG1 was defined as 'no residual tumor cells'), the patients who exhibited an improved response to chemotherapy were associated with decreased expression of HOXC6 (Table II). 


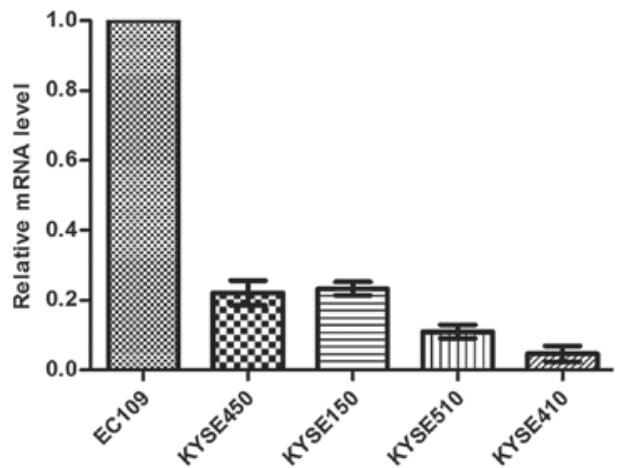

Figure 2. Relative expression of HOXC6 at the mRNA level in the panel of esophageal squamous cell carcinoma cell lines. Basal HOXC6 expression was determined using the reverse transcription-quantitative polymerase chain reaction. EC109 (relatively sensitive cell line) exhibited $\sim 10$-fold upregulation of basal HOXC6 expression compared with relatively resistant cell lines (KYSE510 and KYSE410). Results are expressed as the mean \pm standard deviation relative to EC109 for triplicates from three independent experiments. HOXC6, homeobox C6.

Downregulation of the HOXC6 gene increases ESCC cell chemoresistance. In our previous study (12), the inhibition of cell proliferation induced by chemotherapy agents cisplatin and paclitaxel was determined in a panel of 5 ESCC cell lines (EC109, KYSE450, KYSE510, KYSE410 and KYSE510). It has been demonstrated that EC109 was relatively sensitive, KYSE 410 and KYSE510 were relatively resistant, and KYSE450 and KYSE150 exhibited intermediate sensitivity. Using microarray gene expression profile analysis on these cell lines, a 2.6-fold upregulation of HOXC6 was identified in relatively sensitive ESCC cells, compared with the relatively resistant ESCC cells. The result was confirmed at the mRNA level using qPCR (Fig. 2), and was consistent with results from gene expression profiling. Since HOXC6 was upregulated in relatively sensitive cells, it was hypothesized that HOXC6 may be involved in chemosensitivity. Previously, three small interfering RNAs against HOXC6 were designed to inhibit HOXC6 expression in the intermediately sensitive parental ESCC cell line KYSE450, and stable knockdown cell lines

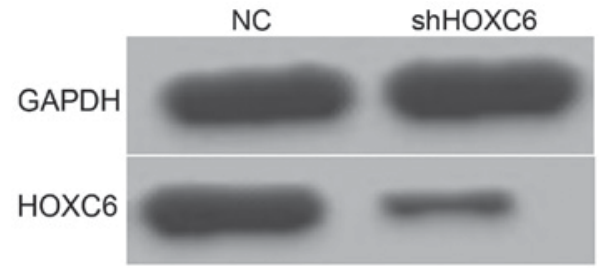

Figure 3. HOXC6 expression at the protein level in esophageal squamous cell carcinoma cell line KYSE450 with knockdown of HOXC6. HOXC6, homeobox $\mathrm{C6}$; NC, negative control; sh, short hairpin.

were established (Fig. 3; data not shown). To investigate the effects of HOXC6 on the chemosensitivity of ESCC, this cell model was used to determine the effect of HOXC6 on cell apoptosis induced by chemotherapy agents. Inhibiting HOXC6 also partially inhibited cisplatin and paclitaxel-induced apoptosis. Treatment for $48 \mathrm{~h}$ with cisplatin and paclitaxel resulted in increased the half-maximal inhibitory concentration $\left(\mathrm{IC}_{50}\right)$ of HOXC6 short hairpin RNA (shRNA)-transfected cells compared with scrambled shRNA-transfected cells (Fig. 4).

\section{Discussion}

In the present study, the expression of HOXC6 was determined using cytology and IHC, and the association between HOXC6 expression and chemosensitivity in ESCC was investigated. The results demonstrated that HOXC6 expression was associated with chemosensitivity in ESCC tissues. Patients with increased expression of HOXC6 prior to treatment were more sensitive to chemotherapy, and knockdown of HOXC6 decreased chemosensitivity to cisplatin and paclitaxel in ESCC cell lines. Thus, determining HOXC6 expression levels prior to surgery may be a predictor of the response to chemotherapy in patients with ESCC.

Oncology, also known as 'disorganized embryology', is associated with the disorder of cell proliferation, death, differentiation, recognition and migration, all of which are involved in embryonic development and cancer. The identification and application of $\alpha$-fetoprotein (AFP) and carcinoembryonic
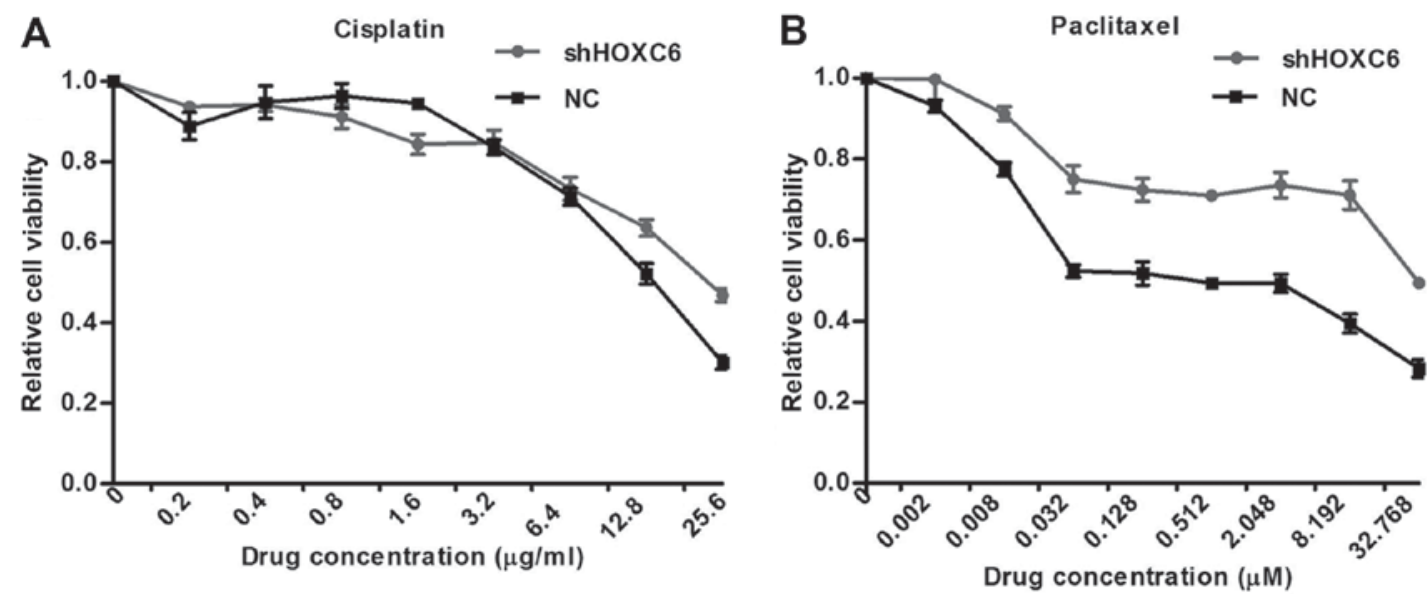

Figure 4. Knockdown of HOXC6 in KYSE450 cells (an intermediately sensitive cell line) increased the cell viability following treatment with chemotherapy drugs (A) cisplatin and (B) paclitaxel. Results are expressed as the mean \pm standard deviation relative to EC109 for triplicates from three independent experiments. HOXC6, homeobox $\mathrm{C} 6$; NC, negative control; sh, short hairpin. 
antigen (CEA) confirmed this view, and suggested that studies of genes and proteins related to embryonic development may be useful for the identification of clinically applicable tumor biomarkers.

As key factors controlling embryonic development, $\mathrm{HOX}$ genes have been demonstrated to be abnormally expressed in a number of types of cancer and are involved in tumor cell proliferation, differentiation and apoptosis. Numerous studies (15-18) have also identified the association between HOX genes and chemosensitivity of tumors. A previous study (19) using gene expression profiling revealed that HOX family members, including HOXA9, HOXA10, HOXB13, HOXC4, HOXC10, HOXC11, HOXC13 and HOXD1, are activated in the temozolomide-resistant pediatric glioblastoma cell line KNS42. Among these activated HOX family members, HOXA9 and HOXA10 are key factors for predicting chemoresistance. Similarly, Tang et al (20) identified that HOXA7, HOXA9, HOXA13, HOXB2, HOXB5, HOXB6, HOXB7, HOXB8, HOXB9 and HOXC9 are overexpressed in the carboplatin-resistant small cell lung cancer cell line DMS53. The expression of HOXA1 in small cell lung cancer tissues is associated with chemoresponsiveness; patients with increased expression of HOXA1 are more sensitive to chemotherapy and thus have improved prognoses compared with patients with decreased expression of HOXA1 (16). Consistently, downregulation of HOXA5 expression in breast cancer cells partly blocks apoptosis induced by retinoic acid-like drugs (21). In chronic myeloid leukemia cells treated with an Abl kinase inhibitor (AMN107) and phosphoinositide 3-kinase inhibitor (LY294002) HOXA10 expression is upregulated, and inhibition of HOXA10 expression, using RNA interference, decreases the sensitivity of chronic myeloid leukemia cells to these two drugs (22). Similarly, HOXB13 overexpression suppresses the synthesis of estrogen receptor (ER) and activates the transcription of interleukin 6 , resulting in resistance to tamoxifen, a commonly used chemotherapeutic drug in patients with $\mathrm{ER}(+)$ breast cancer (23). Therefore, these studies provide important insights into the associations between $H O X$ gene expression and chemoresponsiveness.

In our previous study (12), it was identified that HOXC6 was overexpressed in chemosensitive ESCC cell lines and downregulated in chemoresistant cell lines using a gene expression profiling approach. In the present study, these results were confirmed using qPCR. The influence of HOXC6 on chemosensitivity was further determined using cell lines exhibiting stable knockdown of HOXC6. The results demonstrated that inhibition of HOXC6 expression decreased chemosensitivity and increased the corresponding $\mathrm{IC}_{50}$ values. Therefore, HOXC6 may be associated with chemoresponsiveness in ESCC. To confirm this hypothesis, the expression of HOXC6 was assessed in pretreatment biopsy specimens and matched resected specimens from 51 patients with ESCC who received neoadjuvant chemotherapy. The results demonstrated that patients with higher TRGs exhibited increased expression of HOXC6 in pretreatment biopsy specimens, but no association between TRG and HOXC6 expression was identified in resected specimens. The sample size of resected specimens from patients with neoadjuvant chemotherapy was expanded, and it was revealed that HOXC6 was expressed at decreased levels in postoperative samples from patients with higher
TRGs. Whether HOXC6 may be identified as a potential predictor for evaluating TRG in patients with ESCC therefore remains unclear; however, the results of the present study provide meaningful indications.

There are limitations to the present study. First, the retrospective nature of the study made it difficult to collect pretreatment biopsy specimens and postoperative samples from all patients; therefore, it was not possible to analyze matched tissues in a large sample size. In other words, since it was only possible to analyze the samples that were available, it was not possible to conclusively define the role of HOXC6 in predicting the chemoresponsiveness of ESCC tumors. Larger prospective studies are required to validate the results of the present study.

\section{Acknowledgements}

The authors thank Dr Liang Dai, Dr Xiao-Zheng Kang, Dr Zhen Liang, Dr Hong-Chao Xiong, Dr He-Li Yang and $\mathrm{Dr} \mathrm{Hao} \mathrm{Fu}$ for patient provision and contribution to care. The present study was supported financially by the National Natural Science Foundation of China (grant no. 81301748), the National High Technology Research and Development Program of China (grant no. 2015AA020403), the Beijing Municipal Administration of Hospitals Clinical Medicine Development of Special Funding Support (grant no. ZYLX201509), the National Basic Research Program of China (grant no. 2011CB504300), the Specialized Research Fund for the Doctoral Program of Higher Education (grant no. 20130001110108) and the Science Fund for Creative Research Groups of the Ministry Education of China (grant no. IRT13003).

\section{References}

1. Kelsen DP, Ginsberg R, Pajak TF, Sheahan DG, Gunderson L, Mortimer J, Estes N, Haller DG, Ajani J, Kocha W, et al: Chemotherapy followed by surgery compared with surgery alone for localized esophageal cancer. N Engl J Med 339: 1979-1984, 1998.

2. Medical Research Council Oesophageal Cancer Working Group: Surgical resection with or without preoperative chemotherapy in oesophageal cancer: A randomised controlled trial. Lancet 359: 1727-1733, 2002.

3. van Hagen P, Hulshof MC, van Lanschot JJ, Steyerberg EW, van Berge Henegouwen MI, Wijnhoven BP, Richel DJ, Nieuwenhuijzen GA, Hospers GA, Bonenkamp JJ, et al: Preoperative chemoradiotherapy for esophageal or junctional cancer. N Engl J Med 366: 2074-2084, 2012.

4. Davies AR, Gossage JA, Zylstra J, Mattsson F, Lagergren J, Maisey N, Smyth EC, Cunningham D, Allum WH and Mason RC: Tumor stage after neoadjuvant chemotherapy determines survival after surgery for adenocarcinoma of the esophagus and esophagogastric junction. J Clin Oncol 32: 2983-2990, 2014.

5. Abate-Shen C: Deregulated homeobox gene expression in cancer: Cause or consequence? Nat Rev Cancer 2: 777-785, 2002.

6. Beck F: Homeobox genes in gut development. Gut 51: 450-454, 2002.

7. Grier DG, Thompson A, Kwasniewska A, McGonigle GJ Halliday HL and Lappin TR: The pathophysiology of HOX genes and their role in cancer. J Pathol 205: 154-171, 2005.

8. Chen KN, Gu ZD, Ke Y, Li JY, Shi XT and Xu GW: Expression of $11 \mathrm{HOX}$ genes is deregulated in esophageal squamous cell carcinoma. Clin Cancer Res 11: 1044-1049, 2005.

9. Gu ZD, Shen LY, Wang H, Chen XM, Li Y, Ning T and Chen KN: HOXA13 promotes cancer cell growth and predicts poor survival of patients with esophageal squamous cell carcinoma. Cancer Res 69: 4969-4973, 2009. 
10. Du YB, Dong B, Shen LY, Yan WP, Dai L, Xiong HC, Liang Z, Kang XZ, Qin B and Chen KN: The survival predictive significance of HOXC6 and HOXC8 in esophageal squamous cell carcinoma. J Surg Res 188: 442-450, 2014.

11. Li H, Shen LY, Yan WP, Dong B, Kang XZ, Dai L, Yang YB, Fu H, Yang HL, Zhou HT, et al: Deregulated HOXB7 expression predicts poor prognosis of patients with esophageal squamous cell carcinoma and regulates cancer cell proliferation in vitro and in vivo. PLoS One 10: e0130551, 2015.

12. Shen LY, Wang H, Dong B, Yan WP, Lin Y, Shi Q and Chen KN: Possible prediction of the response of esophageal squamous cell carcinoma to neoadjuvant chemotherapy based on gene expression profiling. Oncotarget 7: 4531-4541, 2016.

13. Mandard AM,DalibardF, Mandard JC, Marnay J,Henry-Amar M, Petiot JF, Roussel A, Jacob JH, Segol P, Samama G, et al: Pathologic assessment of tumor regression after preoperative chemoradiotherapy of esophageal carcinoma. Clinicopathologic correlations. Cancer 73: 2680, 1994

14. Livak KJ and Schmittgen TD: Analysis of relative gene expression data using real-time quantitative PCR and the 2(-Delta Delta C(T)) method. Methods 25: 402-408, 2001.

15. Hamada S, Satoh K, Hirota M, Kanno A, Umino J, Ito H, Masamune A, Kikuta K, Kume K and Shimosegawa T: The homeobox gene MSX2 determines chemosensitivity of pancreatic cancer cells via the regulation of transporter gene ABCG2 J Cell Physiol 227: 729-738, 2012.

16. Xiao F, Bai Y, Chen Z, Li Y, Luo L, Huang J, Yang J, Liao H and Guo L: Downregulation of HOXA1 gene affects small cell lung cancer cell survival and chemoresistance under the regulation of miR-100. Eur J Cancer 50: 1541-1554, 2014.
17. Liu W, Deng L, Song Y and Redell M: DOT1L inhibition sensitizes MLL-rearranged AML to chemotherapy. PLoS One 9: e98270, 2014

18. Li N, Jia X, Wang J, Li Y and Xie S: Knockdown of homeobox A5 by small hairpin RNA inhibits proliferation and enhances cytarabine chemosensitivity of acute myeloid leukemia cells. Mol Med Rep 12: 6861-6866, 2015.

19. Gaspar N, Marshall L, Perryman L, Bax DA, Little SE Viana-Pereira M, Sharp SY, Vassal G,Pearson AD, Reis RM, et al: MGMT-independent temozolomide resistance in pediatric glioblastoma cells associated with a PI3-kinase-mediated HOX/stem cell gene signature. Cancer Res 70: 9243-9252, 2010.

20. Tang CH, Parham C, Shocron E, McMahon G and Patel N: Picoplatin overcomes resistance to cell toxicity in small-cell lung cancer cells previously treated with cisplatin and carboplatin. Cancer Chemother Pharmacol 67: 1389-1400, 2011.

21. Chen H, Zhang H, Lee J, Liang X, Wu X, Zhu T, Lo PK, Zhang X and Sukumar S: HOXA5 acts directly downstream of retinoic acid receptor beta and contributes to retinoic acid-induced apoptosis and growth inhibition. Cancer Res 67: 8007-8013, 2007.

22. Sugimoto Y, Nakamura S, Okinaka K, Hirano I, Ono T, Shigeno K, Shinjo K and Ohnishi K: HOXA10 expression induced by Abl kinase inhibitors enhanced apoptosis through PI3K pathway in CML cells. Leuk Res 32: 962-971, 2008.

23. Shah N, Jin K, Cruz LA, Park S, Sadik H, Cho S, Goswami CP, Nakshatri H, Gupta R, Chang HY, et al: HOXB13 mediates tamoxifen resistance and invasiveness in human breast cancer by suppressing ER $\alpha$ and inducing IL-6 expression. Cancer Res 73: 5449-5458, 2013 